\title{
Design and Implementation of Simulation Operating System for Screw Pump Removal Rod
}

\author{
Xiwen Tong", Haotian Cao, Mingxia Tao and Yuanyuan Jiang \\ Oilfield Exploitation Professional Software Development and Application Project Manager Department of Daqing Oilfield \\ *Corresponding author
}

\begin{abstract}
This paper introduces the use of 3D computer graphics technology, so that the employees can simulate the operation of removing the rod of screw pump in the computer terminal. It avoids the limitation of the site or weather conditions and improves the learning efficiency effectively.

By constructing a 3D model of the oilfield Screw Pump Removal Rod construction site, editing various objects and adding the dynamic behavior of animations and objects, employees can operate as if they were in the field, so as to get familiar with the operation process quickly. Different from the traditional learning method, the intuitive and vivid simulation operation can let the employees quickly learn the theoretical knowledge of screw pump, which makes the foundation for the future field construction.
\end{abstract}

Keywords-3D modeling; behavioral action script; screw pump removal rod

\section{INTRODUCTION}

In the process of oilfield development and construction, the Screw Pump Removal Rod is a part of daily work. When learning and operating, employees often learn through training and onsite observation. This research direction is to simulate the construction site environment, presenting the simulation scene in the form of image on the computer. By tracking the employee's operation behavior, feedback is given to the operation behavior in real time, correct prompts are given to the wrong operation, and the operation is automatically scored to realize human-computer interaction, so that the employees are familiar with the operation skills, safety production, and enhance the employee's business ability.

\section{A. Functional Requirements}

In the virtual three-dimensional scene, the simulation operation of the removal rod can be performed, and a certain score is given according to whether the operation is correct or not,so as to meet the requirements of user operation evaluation.

\section{B. Technical Ideas}

The template is designed so that author affiliations are not repeated each time for multiple authors of the same affiliation. Please keep your affiliations as succinct as possible (for example, do not differentiate among departments of the same organization). This template was designed for two affiliations.

1) Use 3DS MAX (3D animation rendering and production software) to build 3D scene models, including sky and earth, screw pump, and construction equipment.
2) Use Virtools (real-time 3D environment virtual reality editing software) to create real-time dynamic scripts, give image model action behavior, and respond to various operations, including roaming scenes, mouse following, device picking, error handling and score calculation.

\section{DESIGN AND IMPLEMENTATION}

\section{A. 3D Scene Model Construction}

The 3D image model of screw pump well and sky and earth is constructed in 3DS MAX, and the lighting is adjusted. Finally, the 3D scene model is imported into Virtools in NMO format. If any problem was found in the scene, it is directly adjusted in Virtools to show the best effect of the scene.

\section{1) Construction Scenario}

In 3DS MAX, the three-dimensional image model of the screw pump well is divided into individual components for graphic processing, including pipes, valves, motors, large fourway, polished rods, polished rod plugs, slips, lifting short sections, elevators, and fixed clips, small hooks, pipe wrenches, wrenches, socket wrenches, and finally these parts are combined to form the screw pump well model.

The sky and earth three-dimensional image model adopts ellipsoidal figure. Set the sphere radius, select the sky and earth bitmap, assigns the sky and earth material to the ellipsoid, and performs mapping processing[1].

\section{2) Lighting debugging}

Place the light source in the 3D image model of the sky and earth, adjust the position above the sky to illuminate the sky and earth, and adjust the intensity and illumination range of the light.

Combine the screw pump well and the three-dimensional image model of the sky and earth, and import them into Virtools.

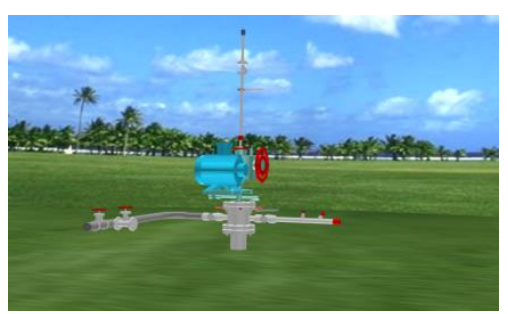

FIGURE I. THREE-DIMENSIONAL IMAGE MODEL OF SCREW PUMP WELL 


\section{B. Maintaining Functional Design}

The simulation operation evaluation system of Screw Pump Removal Rod includes device identification, error handling, interaction, roaming scene, scoring calculation and other functions. The function description is as follows:

1) Device identification: click the mouse to trigger device identification, determine whether the $3 \mathrm{~d}$ image model is a pipe wrench, a polished rod or an elevator, and then execute the response action script.

2) Error handling: It is whether the $3 \mathrm{D}$ image model recognized by the processing device is in the component device list, and the error message is automatically prompted, and the score of this step is recorded as 0 . Otherwise, error handling is not triggered.

3) Roaming scene: One is the free perspective, the controller can freely adjust the perspective of the scene; The other is the operating perspective, which has been set and cannot be adjusted by the controller.

4) Score calculation: There are two types of score, 0 points and 8 points. If the operation is correct, it is recorded as 8 points. If the operation is wrong, it is recorded as 0 . According to the operation of each step, the final score of this step is determined.

5) Interactive operation: It is divided into seven operation steps. Step 1: Selecting the pipe clamp and unloading the light bar. Step 2: Select the lifting short section and install it on the polished rod. Step 3: install the elevator, small hook. Step 4: Remove the fixed clip. Step 5: Move down to raise the short section. Step 6: Remove the lifting nipple. Step 7: Remove the big four links.

\section{System Implementation}

The 3D model of the Screw Pump Removal Rod construction site is completed, but they are all static images. It is necessary to conduct behavioral programming on the static images in Virtools, so that the system can automatically perform responsive behaviors according to the operational events of the controller. To this end, a message control module needs to be established, and the message control module receives the operation message and gives a corresponding operation[2].

The type of receiving message includes, firstly, receiving a keyboard message, and the result of the message response is that the angle of view is translated and rotated with the keyboard instruction. Secondly, the receiving tool picks up the message, and the result of the message response is that the tool automatically follows the mouse movement. Thirdly, receiving the device identification message, the system determines whether the device identified by the current operation step is in the list of executable action components, and the message response result is that the component action script is automatically executed. If the recognition fails, an operation error script is executed to give a correct operation prompt , and the current operation score results.

Establish a operation step table, record the score of each step operation, prompt information and the components need to identify each step. Establish a correspondence between each step operation and the component action script. When the controller performs each step of operation, the system automatically goes to the step table to perform identification information, prompt information, and score information processing, and feeds back to the controller.

1) Roaming Scene: Tracking the mouse and keyboard events operated by the controller, and sets the translation unit or rotation angle of each operation step to achieve the perspective of translation and rotation. This function realizes the perspective moving up 5 units, down 5 units, left 5 units, right 5 units, left 15 degrees rotation, right 15 degrees rotation. Building Blocks required: Switch On Way, Per Second, Translate, Rotate.

2) Device Identification and Error Handling: When clicking a part of the screw pump well model, the system picks up the part information, the system searches the operation step table, determines whether the part is in the device list, and if so, executes the corresponding action script, if not going to the error processing, the error is displayed. Then prompt for another operation until the operation prompts the next step. The required building blocks include Switch On Message, Mouse Waiter, 2D Picking, Row Search, Get Cell, Is In Group, Set cell, Send Message, Delayer, and Text Display.

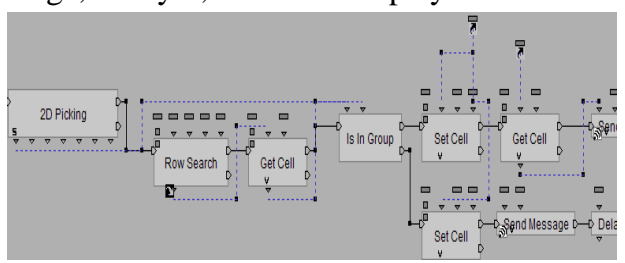

FIGURE II. DEVICE IDENTIFICATION SCRIPT

3) Score calculation: Set the score of each step to 8 and if the operation is wrong, it will automatically change the singlestep score to 0 . Otherwise, the single-step score will remain unchanged at 8. In this way, after all the actions are completed, all the single-step scores are added, and the final score is the total score of the entire operation, and then the single-step score and the total score are displayed.

\section{CONCLUSION}

Through the research and application of the simulation and operation of the Screw Pump Removal Rod construction, the employees have an immersive experience in operation training, and explore the new direction of the deep application of 3D computer graphics technology. The simulation operation has a good application prospect in the oilfield.

\section{REFERENCES}

[1] Lianxing Jia, Yinghao Zhu, Jiang Zhang. "3D Visual Simulation[M]. Beijing: " National Defense Industry Press, 2017.

[2] Junshan Li, Rui Wang, Jianjun Li. "3D Scene Simulation and Visual Modeling[M]. Beijing: " Science Press, 2011 